\title{
DIPOLE-PROTON $S$-MATRIX DERIVED FROM DIFFRACTIVE MESON ELECTROPRODUCTION
}

\author{
S. MUNIER \\ INFN, Sezione di Firenze, Sesto Fiorentino (Firenze), 50019, Italy.
}

\begin{abstract}
Through a determination of the $S$-matrix element for the scattering of a localized colour dipole on a proton, we show that saturation effects can already be sizable at HERA. The saturation scale is found to be around $1-1.5 \mathrm{GeV}^{2}$ for the highest available energies and for central collisions, and is a decreasing function of the impact parameter.
\end{abstract}

Presently, there are basically two most discussed theoretical descriptions of the HERA data for the proton structure functions, equally successful at small- $x$ but contradictory in their physical implications. On one hand, models based on the DGLAP equation fit the data very well. On the other hand, the recent saturation modell reproduces all small- $x$ data with a small number of parameters. The latter assumes a unitarized cross section but no scale evolution is taken into account. Its success could mean that saturation effects are already important at HERA, at a scale around $1 \mathrm{GeV}^{2}$. However, the very question whether we have reached or not the regime of high densities at HERA in the perturbative domain has so far not been addressed in a direct way. We propose here a simple method to determine reliably how dense the proton looks.

\section{A brief introduction to saturation}

The HERA physics in the kinematical range considered here is the physics of photon-proton interactions. We will denote $W$ the $\gamma^{*} p$ center-of-mass energy, $Q^{2}$ the virtuality of the photon and $x \simeq Q^{2} / W^{2}$ the Bjorken variable. In a Bjorken frame in which the proton has a large light-cone momentum $\left(p^{+}, 0, \mathbf{0}\right)$ and the photon the momentum $\left(0, q^{-}, \mathbf{q}^{\perp}\right)$, the photon-proton interaction can be interpreted as the photon probing the partonic content of the proton. This probe is sensitive to collinear fluctuations down to transverse distances of order $1 / q^{\perp} \propto 1 / Q$, and resolves fluctuations occuring over times as short as $1 / q^{-} \propto$ $p^{+} / W^{2}$. Thus by increasing $Q^{2}$, one reveals more collinear partons. By raising the center of mass energy $W$, one becomes sensitive to softer fluctuations.

If one keeps increasing $W$ at fixed $Q$, the density of partons of size $1 / Q$ will eventually be so large that their wave functions will overlap, and recombination effects will become sizable. The borderline between these two regimes

proceedings: submitted to World Scientific on October 26, 2018 
is usually parametrized by the function $Q^{2}=Q_{s}^{2}\left(W^{2}\right) . Q_{s}$ is called the "saturation scale". The question is whether we have already crossed this line at HERA.

Generally speaking, wave diffraction allows to obtain a picture of a microscopic object. A mere Fourier transform then relates the diffractive pattern to the density profile of the object. This can be done at HERA using diffractive processes, as will be discussed in the following.

\section{Transparency of the proton to dipoles from diffraction}

At high energy, one can relate diffractive vector meson electroproduction to dipole-proton elastic scattering. This is the celebrated dipole modele, that we briefly recall here.

In the proton rest frame, the scattering proceeds in three steps. The virtual photon first breaks up into a $q \bar{q}$ pair of size $\mathbf{r}$, which then scatters off the proton, before being recombined into a meson. High $W$ guarantees that these processes are well separated in time. At HERA, the $q \bar{q}$ pair travels over distances of a few $10^{2}$ fermis before entering the interaction region, of size 1 $\mathrm{fm}$. The recombination takes a comparable "time". This remark leads to the following factorization formula for the amplitude $\mathcal{A}$ of this process:

$$
\mathcal{A}\left(Q^{2}, W^{2}, \Delta\right)=\int d^{2} \mathbf{r} A\left(W^{2}, \mathbf{r}, \Delta\right) \int d z \psi_{Q}(z, \mathbf{r}) \psi_{V}^{*}(z, \mathbf{r}),
$$

where $A$ is the dipole-proton elastic amplitude, $\psi_{Q}$ and $\psi_{V}$ are the photon and vector meson wave functions respectively. The latter has to be modelled: we considered several models available in the litterature. $\Delta$ is the 2-momentum transfer: it is conjugated to the impact parameter, and this will be the variable with respect to which we will take the Fourier transform. We define $t \equiv|\Delta|^{2}$.

Thus the proton sees a beem of asymptotic $q \bar{q}$ dipoles, distributed according to the overlap of the photon and meson wave functions (second integral in formula (1)). On very general grounds, one can argue that this function is sharply peaked around a value $r_{Q}$ of the dipole size scaling like $1 / \sqrt{Q^{2}+M_{V}^{2}}\left(M_{V}\right.$ is the mass of the meson, see 2). Hence the amplitude can be approximated by $\mathcal{A}\left(Q^{2}, W^{2}, \Delta\right) \simeq A\left(W^{2}, r_{Q}, \Delta\right) \times N(Q)$ where $N(Q)$ is the normalization of the dipole distribution. Note that $N(Q)$ is a priori model-dependent, but in practice this dependence is very weak.

Once we have extracted the dipole-proton amplitude from the HERA data in this way, we perform a Fourier analysis, by defining the function

$$
S\left(W^{2}, r_{Q}, b\right)=1-\frac{1}{2} \int \frac{d^{2} \Delta}{(2 \pi)^{2}} e^{-i \mathbf{b} \Delta} A\left(W^{2}, r_{Q}, \Delta\right) .
$$

proceedings: submitted to World Scientific on October 26, 2018 
One easily checks that $S\left(W^{2}, r, b\right)$ is the $S$-matrix element for the scattering of a localized dipole of given size $r$ at given impact parameter $b$. This holds in particular because at high energy such a dipole state can only be absorbed: the $S$-matrix is diagonal and its eigenvalues are real between 0 and 1 . They can be interpreted as kind of "transparency coefficients". Furthermore, note that $1-S^{2}\left(W^{2}, r, b\right)$ is the probability for such a dipole state to undergo inelastic scattering.

\section{A picture of the proton at HERA and its interpretation}

We have applied the procedure described above to the HERA data. We took the available data for diffractive production of longitudinal $\rho$ mesons from the H1 collaborationl. $x$ was taken small, around $5 \cdot 10^{-4}$. Three different values of $Q^{2}$ were considered, $Q^{2}=7,3.5$, and $0.45 \mathrm{GeV}^{2}$, corresponding to dipole sizes of about $0.16,0.21$ and $0.35 \mathrm{fm}$ respectively. The data was available only for $t<0.6 \mathrm{GeV}^{2}$, so we had to extrapolate them to larger values of $\Delta$ before performing the Fourier transform. We made three such assumptions. The results are represented on fig. 1. We get a reliable extraction of $S(b)$ down to $b=0.3 \mathrm{fm}$.

We see that for say $Q^{2}<2 \mathrm{GeV}^{2}$ and $b<0.4 \mathrm{fm}$, the probability of inelastic interaction is more than $50 \%$. Hence the proton looks already quite black in its central region at present HERA energies. To see whether this "blackening" occurs in the perturbative regime, we estimate the saturation scale. For this purpose, it is necessary to choose a parametrisation for $S$. Let us consider the following Glauber-like form:

$$
S\left(W^{2}, r, b\right)=e^{-Q_{s}^{2}\left(W^{2}, b\right) r^{2} / 4},
$$

where the fact that $Q_{s}$ controls the onset of the saturation regime is quite explicit. From this formula we now extract the saturation scale and obtain $Q_{s}^{2} \simeq 1-1.5 \mathrm{GeV}^{2}$ for $b=0.3 \mathrm{fm}$ and $Q_{s}^{2} \simeq 0.2 \mathrm{GeV}^{2}$ for $b=1.0 \mathrm{fm}$.

We refer the reader to the paper 6 for all the details of this analysis and for more results.

\section{Acknowledgments}

The results presented here were obtained in collaboration with Dr A.M. Staśto and Pr A.H. Mueller. I thank Dr J.-Y. Ollitrault for a critical reading of the manuscript. I also wish to acknowledge support from the EU Framework TMR program, contract FMRX-CT98-0194.

proceedings: submitted to World Scientific on October 26, 2018 


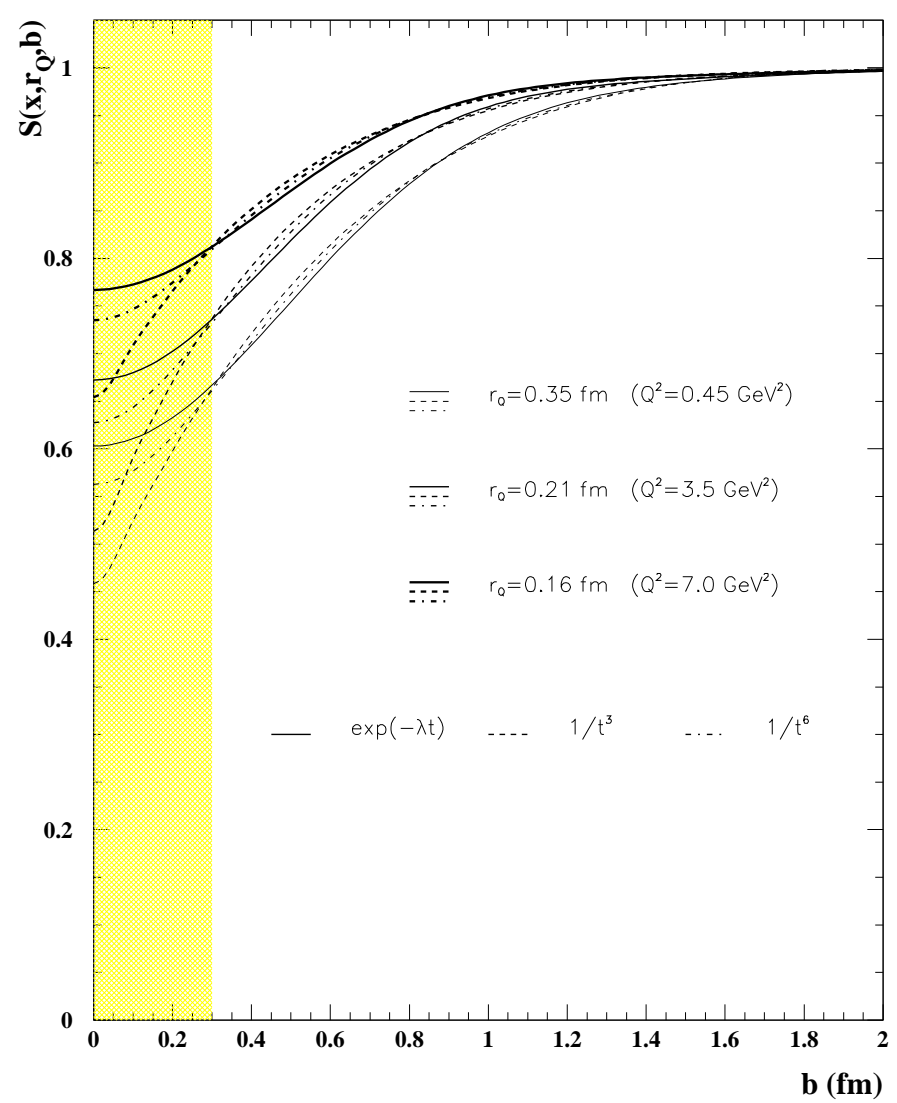

Figure 1. $S$-matrix element as a function of $b$, for $x=5 \cdot 10^{-4}$. Three values of $Q^{2}$ are considered, and three different extrapolations of the data at large momentum transfer $\left(\exp (-\lambda t), t^{-3}, t^{-6}\right)$. The sensitivity to this extrapolation is high in the grey zone.

\section{References}

1. K. Golec-Biernat, M. Wüsthoff, Phys. Rev. D59 (1999) 014017.

2. See J. Nemchik, N.N. Nikolaev, B.G. Zakharov, Phys.Lett. B341 (1994) 228 and references therein.

3. H1 collaboration Eur. Phys. J. C13 (2000) 371.

4. S. Munier, A. M. Staśto, A. H. Mueller, Nucl. Phys. B603 (2001) 427. 